\title{
Discovering the complexity of the metazoan transcriptome
}

\author{
Matthew J Hangauer, Susan Carpenter and Michael T McManus ${ }^{*}$
}

\author{
Abstract \\ Recent studies harnessing deep RNA sequencing \\ coupled with other complementary data have \\ revealed the complex nature of metazoan \\ transcriptomes.
}

\section{The deeper you look, the more you find: pervasive transcription}

The advent of deep sequencing technology has ushered in a new era of genomics research. The application of deep sequencing to RNA analysis has shown that the majority of the genomic sequence is transcribed in humans and other metazoan species [1]. These analyses have resulted in the identification of thousands of novel coding and non-coding genes. Yet, it is clear that the full catalog of transcription remains elusive. For example, when presumed non-transcribed human intergenic sequence was studied in great depth, a complex web of overlapping, spliced transcripts of very low abundance was found [2]. These and other findings indicate that the complexity of the transcriptome is currently underappreciated.

In 2012, as part of the ENCODE project, which focused initially on humans, Djebali et al. [1] showed the staggering complexity and expanse of the human transcriptome. At least three-quarters of the genome produces transcripts, many of which demonstrate complex splicing and specific expression patterns. Recent results from the modENCODE project by Brown et al. [3] analyzing the Drosophila transcriptome have shown similarly impressive complexity and some surprising differences compared with humans.

\section{Digging even deeper: splicing}

The ENCODE project team observed that human genes do not follow a minimalistic isoform expression strategy;

\footnotetext{
* Correspondence: michael.mcmanus@ucsf.edu

Diabetes Center, Department of Microbiology and Immunology, University of California, San Francisco, CA 94143, USA
}

instead each gene simultaneously produces up to 10 to 12 isoforms [1]. In contrast, Brown et al. [3] observed that in Drosophila, most genes have relatively few isoforms. In fact, $42 \%$ of Drosophila genes encode only a single transcript isoform, whereas in mammals, $95 \%$ of genes have multiple expressed isoforms [1,4]. This difference in number of isoforms per gene is unlikely to be due to uneven sampling or read depth between humans and Drosophila. The Drosophila data consist of over 12 billion read pairs from many tissues and conditions, with enough read depth and conditions to identify isoforms of most genes, and are at least on a par with the read depth of the human data. Interestingly, in Drosophila, the majority of alternatively spliced genes encode only a single protein sequence and vary only in the first exon, through either alternative promoter usage or splicing [3]. Genome-wide analysis of human isoform proteincoding capacity has not been reported, and it will be interesting to determine whether human alternative splicing significantly affects the protein sequence.

Splicing in Drosophila was found to be highly tissuespecific, with over $50 \%$ of splicing events changing significantly between tissues [3]. Tissue-specific splicing appears to be more prevalent than sex-specific or developmental stage-specific splicing. In fact, sex-specific splicing was only found in sex tissues present exclusively in males or females. The underlying reasons for this dominance of tissue-specific splicing in Drosophila are unclear, but it may be due to a variety of causes, including the strongly tissue-specific expression or regulation of different components of splicing machinery.

Though Brown et al. [3] found that Drosophila genes have fewer isoforms than human genes, in general, they observed very complex splicing of some Drosophila genes. Specifically, they found that 47 genes each have an extremely high number (more than 1,000) of splice variants and are expressed primarily in developing and adult neural Drosophila tissue. These transcripts are strongly enriched for RNA-editing, possessing 3' UTR extensions, and the total count of these genes' isoforms makes up half 
of all transcripts in Drosophila. The biological significance of the extreme splicing of these genes is unclear, and it is currently unknown whether orthologs of these genes have similar splicing patterns in other organisms.

\section{Venturing into the dark matter of the genome}

Utilizing extensive RNA-seq data, Brown et al. [3] observed that, similar to humans, both antisense and intergenic non-coding transcription is prevalent in Drosophila. In particular, Drosophila gonad tissue was found to express many previously unknown transcripts, consistent with observations in testes from other species [5,6]. Indeed, it has been hypothesized that testes tissue has a permissive chromatin environment allowing transcription, perhaps serving as a gene creation tissue during evolution [6].

Most antisense transcription in Drosophila results from overlapping mRNAs on the opposite strand and the majority is the result of overlapping UTRs. However, $21 \%$ of Drosophila long noncoding RNAs (lncRNAs) are antisense to mRNAs (compared with 15\% for humans) and some lncRNAs overlap multiple mRNAs forming 'sense-antisense gene-chains', similar to those seen in mammals [7]. The degree to which sense and antisense transcripts are present in the same cells, compared with mutually exclusive expression within distinct cells in a cell population, is unclear and is an important avenue for future research.

Earlier studies in Drosophila have catalogued lncRNAs using non-stranded RNA-seq data of limited read depth and tissue breadth. Using a vast set of stranded RNA-seq data, Brown et al. [3] found up to 4,000 candidate IncRNA genes with no predicted open reading frame (ORF) longer than 100 amino acids. Further refinement of this catalog, including removal of putative conserved short protein-coding genes, resulted in a catalog of about 2,000 lncRNAs. In comparison, there are 13,870 human lncRNA genes annotated in GENCODE v19. Due to the low expression of most lncRNAs and poor read coverage, Brown et al. catalogued the full structures of only a few hundred Drosophila lncRNAs. In comparison, human lncRNAs discovered from RNA-seq and other complementary deep sequencing data also suffer from incomplete structures $[5,8]$. As a result, it is unclear whether the different total IncRNA count in humans and Drosophila reflects actual lncRNA numbers or is an artifact of incomplete annotation. In addition, Brown et al. only studied polyadenylated RNA, so it is possible that there are many nonpolyadenylated lncRNAs that remain unidentified: the overall complexity of the Drosophila non-coding transcriptome could currently be underestimated.

Putative lncRNAs that have not been experimentally evaluated for protein-coding capacity could be novel protein-coding genes. High throughput refinement of candidate lncRNAs to remove very short peptideencoding genes, such as the Drosophila gene tarsel-less (11 amino acids), is currently not technically feasible. Ribosomal profiling data, if available across all tissues of interest, could be used to identify only those transcripts that have no physical association with the ribosome. However, recent studies have shown that lncRNAs can associate with the ribosome yet not encode a protein [9]. Furthermore, mass spectrometry has been used effectively to identify some small peptide-encoding genes [10]. Unfortunately, mass spectrometry is prone to false negatives and therefore is not a reliable method to identify all short peptide-encoding genes exhaustively, a requirement if it were to be used as a robust filter for lncRNAs. As a result, there is currently no high throughput empirical or computational approach to definitively segregate non-coding from coding transcripts with absolute confidence. This is a major challenge for the lncRNA field and new approaches are needed.

Despite the incompleteness of lncRNA annotations, it is nonetheless clear that lncRNAs are frequently present within non-coding regions of the genome known to be functional. IncRNAs are strongly enriched for traitassociated single nucleotide polymorphisms (SNPs) in humans [8]. In Drosophila, of all the novel coding and noncoding genes Brown et al. discovered, only lncRNAs overlap previously molecularly defined mutations with phenotypes [3]. This evidence, as well as a growing list of examples of functional human lncRNAs containing trait-associated SNPs (such as MIAT), has supported the possibility that much of the intergenic functional sequence in organisms acts through non-coding RNA rather than, or in addition to, DNA. However, it remains to be experimentally determined what fraction of, and which, lncRNAs act as functional transcripts.

\section{Where are we going and how do we get there?}

Deep sequencing technology has allowed for an unprecedented view of transcriptomes, revealing tremendous complexity in transcript identity, splicing and expression patterns. Complementary techniques, including RNAseq, CAGE, 5' and $3^{\prime}$ end sequencing and others have provided large amounts of detailed information. The recent studies described here from the ENCODE and modENCODE projects exemplify what can be learned from the application of deep sequencing approaches to transcriptomes (Figure 1).

Despite this impressive array of technologies and large-scale collaborative efforts to apply them to transcriptomes, a fully comprehensive catalog of any organism's transcriptome remains elusive. Indeed, even the complete saturation of sampling of all RNA species within a single cell type has yet to be accomplished. 


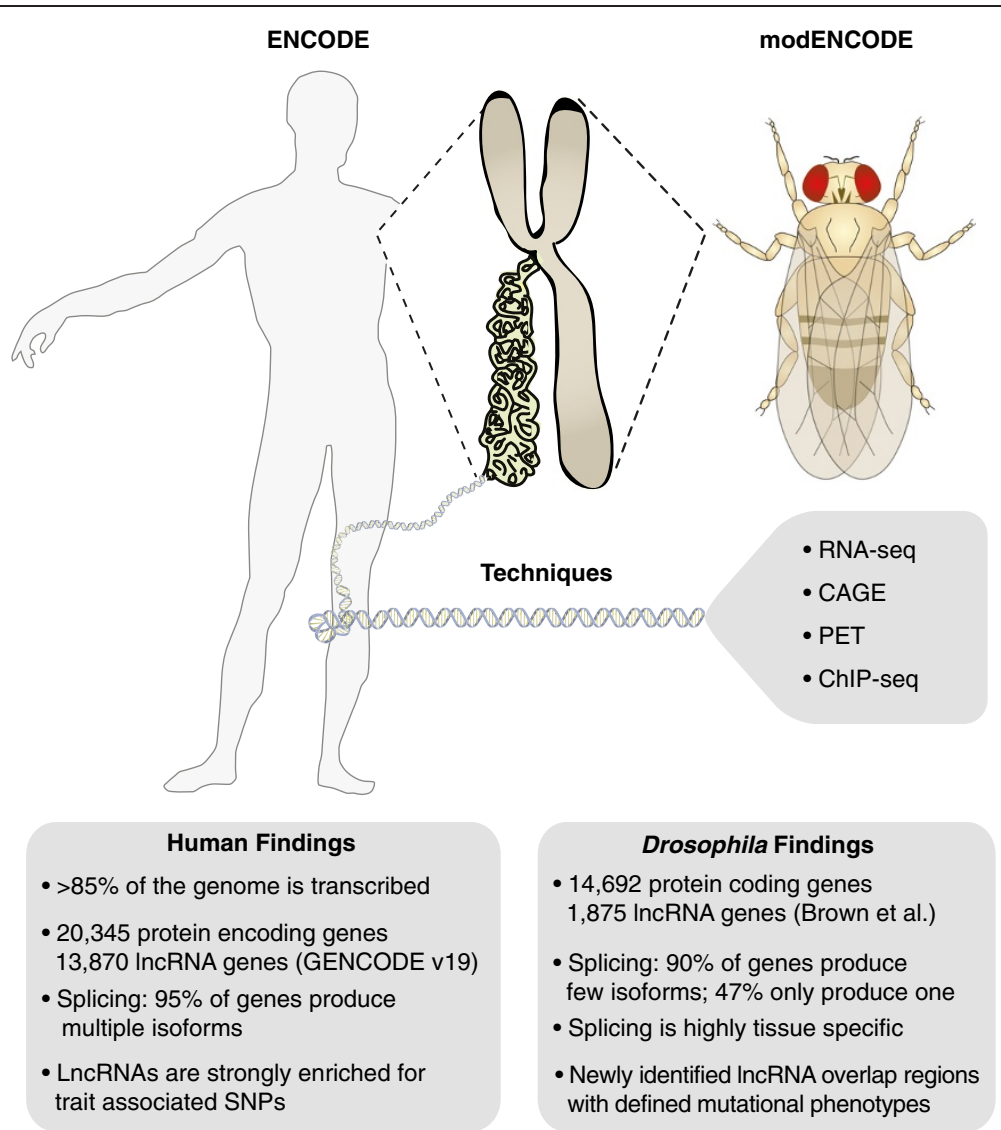

Figure 1 Discovering the complexities of human and Drosophila transcriptomes. The ENCODE (left-hand side) and modENCODE (right-hand side) projects have used a diverse set of techniques to provide an unprecedented view of the human and Drosophila transcriptomes.

Many technical hurdles remain. First and foremost, what read depth is required to saturate sampling of all transcripts? The discovery of very low abundance transcripts illustrates how difficult it may be to answer this question [2]. Can a transcript with apparent expression in 1 out of 10 cells be functional? 1 out of 100 ? 1 out of 1,000 ? Is there meaningful biological information in many or few of these low abundance transcripts? Determining the limits of transcript abundance for functionality will be important in directing future efforts toward a full understanding of the transcriptome.

There are many additional technical challenges on the horizon. RNA transcripts may be capped or not, polyadenylated or not, vary drastically in size from a few nucleotides to megabases, and overlap each other in complex ways. Current deep sequencing technology does not allow for very long read lengths and extremely deep read depths at an affordable cost, yet this may be the only approach to unequivocally define full transcript structures, particularly of lowly expressed transcripts in complex loci. Another challenge on the horizon is the analysis of single cell transcriptomes. Due to difficulties in reliably isolating single cell RNA from tissues and robustly sequencing RNA from a single cell, the amount and biological importance of single cell heterogeneity of RNA expression remains controversial.

Despite these challenges, the studies by Brown et al. [3] and Djebali et al. [1] have provided important insight into the nature of the Drosophila and human transcriptomes. These studies and others to come will serve as fundamental resources for increasing our understanding of the transcriptome in its complicated beauty.

\section{Abbreviations \\ IncRNA: long noncoding RNA; SNP: single nucleotide polymorphism; \\ UTR: untranslated region.}

\section{Competing interests}

The authors declare that they have no competing interests.

Published: 29 April 2014

\section{References}

1. Djebali S, Davis CA, Merkel A, Dobin A, Lassmann T, Mortazavi A, Tanzer A, Lagarde J, Lin W, Schlesinger F, Xue C, Marinov GK, Khatun J, Williams BA, Zaleski C, Rozowsky J, Röder M, Kokocinski F, Abdelhamid RF, Alioto T, Antoshechkin I, Baer MT, Bar NS, Batut P, Bell K, Bell I, Chakrabortty S, Chen $X$, Chrast J, Curado J, et al: Landscape of transcription in human cells. Nature 2012, 489:101-108. 
2. Mercer TR, Gerhardt DJ, Dinger ME, Crawford J, Trapnell C, Jeddeloh JA, Mattick JS, Rinn JL: Targeted RNA sequencing reveals the deep complexity of the human transcriptome. Nat Biotechnol 2013, 30:99-104.

3. Brown JB, Boley N, Eisman R, May GE, Stoiber MH, Duff MO, Booth BW, Wen J, Park S, Suzuki AM, Wan KH, Yu C, Zhang D, Carlson JW, Cherbas L, Eads BD, Miller D, Mockaitis K, Roberts J, Davis CA, Frise E, Hammonds AS, Olson S, Shenker S, Sturgill D, Samsonova AA, Weiszmann R, Robinson G, Hernandez J, Andrews J, et al: Diversity and dynamics of the Drosophila transcriptome. Nature 2014. doi: 10.1038/nature12962 [Epub ahead of print].

4. Wang ET, Sandberg R, Luo S, Khrebtukova I, Zhang L, Mayr C, Kingsmore SF, Schroth GP, Burge CB: Alternative isoform regulation in human tissue transcriptomes. Nature 2008, 456:470-476.

5. Cabili MN, Trapnell C, Goff L, Koziol M, Tazon-Vega B, Regev A, Rinn JL: Integrative annotation of human large intergenic noncoding RNAs reveals global properties and specific subclasses. Genes Dev 2011, 25:1915-1927.

6. Soumillon $M$, Necsulea $A$, Weier $M$, Brawand $D$, Zhang $X, G u H$, Barthès $P$, Kokkinaki M, Nef S, Gnirke A, Dym M, de Massy B, Mikkelsen TS, Kaessmann $\mathrm{H}$ : Cellular source and mechanisms of high transcriptome complexity in the mammalian testis. Cell Rep 2013, 3:2179-2190.

7. Katayama S, Tomaru Y, Kasukawa T, Waki K, Nakanishi M, Nakamura M, Nishida H, Yap CC, Suzuki M, Kawai J, Suzuki H, Carninci P, Hayashizaki Y, Wells C, Frith M, Ravasi T, Pang KC, Hallinan J, Mattick J, Hume DA, Lipovich L, Batalov S, Engström PG, Mizuno Y, Faghihi MA, Sandelin A, Chalk AM, Mottagui-Tabar S, Liang Z, Lenhard B, et al: Antisense transcription in the mammalian transcriptome. Science 2005, 309:1564-1566.

8. Hangauer MJ, Vaughn IW, McManus MT: Pervasive transcription of the human genome produces thousands of previously unidentified long intergenic noncoding RNAs. PLoS Genet 2013, 9:e1003569.

9. Guttman M, Russell P, Ingolia NT, Weissman JS, Lander ES: Ribosome profiling provides evidence that large noncoding RNAs do not encode proteins. Cell 2013, 154:240-251.

10. Slavoff SA, Mitchell AJ, Schwaid AG, Cabili MN, Ma J, Levin JZ, Karger AD, Budnik BA, Rinn JL, Saghatelian A: Peptidomic discovery of short open reading frame-encoded peptides in human cells. Nat Chem Bio/ 2013, 9:59-64.

doi:10.1186/gb4172

Cite this article as: Hangauer et al:: Discovering the complexity of the metazoan transcriptome. Genome Biology 2014 15:112. 